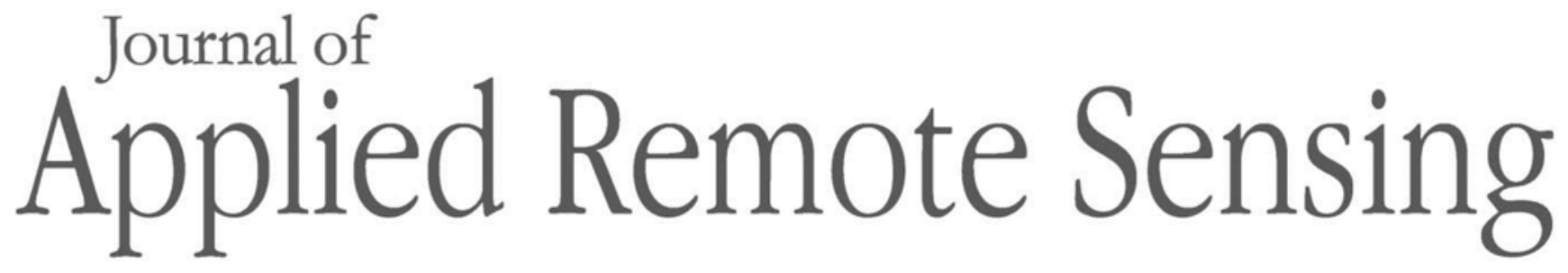

RemoteSensing.SPIEDigitalLibrary.org

\title{
Validation of a ground-based telescope-assisted hyperspectral remote sensor for soil measurements
}

Sean E. Salazar

Richard A. Coffman 


\title{
Validation of a ground-based telescope-assisted hyperspectral remote sensor for soil measurements
}

\author{
Sean E. Salazar ${ }^{\mathrm{a}, *}$ and Richard A. Coffman ${ }^{\mathrm{b}}$ \\ ${ }^{a}$ Norwegian Geotechnical Institute, Oslo, Norway \\ ${ }^{b}$ University of Arkansas, Department of Civil Engineering, Fayetteville, \\ Arkansas, United States
}

\begin{abstract}
The utility of the ground-based soil observation laser absorption spectrometer (SOLAS) was demonstrated through spectral reflectance measurements of five soil types during laboratory- and field-based collection. The SOLAS telescope-assisted measurements were compared with proximal measurements. The spectra that were acquired at an intermediate range of 40 meters compared well with the spectra that were acquired proximally. Specimen type, rangedependent spatial resolution, and environmental conditions are discussed. The signal-to-noise ratio (SNR) was assessed and is presented as a function of wavelength for the spectral range of the receiver for each measurement condition. The proximal measurements performed outdoors under solar illumination had the greatest SNR, while the remote measurements performed indoors under artificial illumination had the lowest SNR. For the outdoor measurements, loss of signal was observed around the 1400- and 1900-nm bands due to long-path atmospheric water vapor absorption. The discussed future improvements to the SOLAS remote sensor will enable measurements of reflectance over longer ranges. Envisioned applications include remote characterization of surface materials for large construction projects (e.g., surface mines and tailings) for geohazard investigations, or for ground truthing of current and future multispectral and hyperspectral satellite data. (c) The Authors. Published by SPIE under a Creative Commons Attribution 4.0 Unported License. Distribution or reproduction of this work in whole or in part requires full attribution of the original publication, including its DOI. [DOI: 10.1117/1.JRS.14.027503]
\end{abstract}

Keywords: instrument development; hyperspectral; reflectance; spectroradiometry; telescope; soil.

Paper 190527 received Jul. 9, 2019; accepted for publication Apr. 22, 2020; published online May 14, 2020.

\section{Introduction and Background}

Hyperspectral sensing, primarily in the visible near-infrared (VNIR) and shortwave infrared (SWIR) ranges, has become increasingly utilized for ecologic ${ }^{1}$, geologic, ${ }^{2}$ civil engineering, ${ }^{3}$ and agronomic ${ }^{4}$ applications. While high spectral resolution $(\leq 10 \mathrm{~nm})$ sensing for rapid characterization of rocks, minerals, soils, and vegetation is commonly performed at a proximal range $(<1 \mathrm{~m})$, remote measurements with increasingly higher spectral and spatial resolutions have been demonstrated. ${ }^{5,6}$ The proliferation of compact sensors has enabled portable and accurate measurements of spectral reflectance from ground-based, manned and unmanned aircraft, and spaceborne platforms. There is continued interest in developing hyperspectral remote sensing technologies for a variety of applications, for example, for land-use/land-cover classification ${ }^{7}$ with several countries investing in planned space missions. ${ }^{8}$ The prototype soil observation laser absorption spectrometer (SOLAS), which is discussed in this paper, enabled high spectral resolution measurements at ranges greater than conventional proximal measurements while maintaining cost and deployment feasibility. This type of device is needed because previous airborne missions have been cost prohibitive and continuous full-spectrum sensors have only recently overcome payload limitations for heavy unmanned platforms. ${ }^{9,10}$ Deployment of the SOLAS could support remote characterization of rocks, minerals, soils, or vegetation on inaccessible slopes or outcroppings for economic geology, earthworks, or agriculture and forestry research

*Address all correspondence to Sean E. Salazar, E-mail: sean.salazar@ngi.no 
applications. The SOLAS could also provide a means for ground truthing data collected from current and future multispectral and hyperspectral missions.

The development of the SOLAS prototype was described previously in the literature. ${ }^{11,12}$ In its current form, the SOLAS utilizes a portable, tripod-mounted telescope that collimates reflected light through an optical receiver into a high radiometric-resolution spectroradiometer [Analytical Spectral Devices (ASD) FieldSpec 4 Hi-Res; Malvern Panalytical Ltd., Malvern, United Kingdom]. Light is detected on 2151 continuous bands from the ultraviolet to SWIR wavelengths. The FieldSpec instrument utilizes a silicon detector for the VNIR wavelength range ( 350 to $1000 \mathrm{~nm}$ ) with a spectral resolution of $3 \mathrm{~nm}$ and a sampling interval of $1.4 \mathrm{~nm}$. A pair of thermoelectric-cooled indium gallium arsenide (InGaAs) detectors provide a spectral resolution of $8 \mathrm{~nm}$ and a sampling interval of $1.1 \mathrm{~nm}$ for the SWIR wavelength ranges from 1001 to $1800 \mathrm{~nm}$ and 1801 to $2500 \mathrm{~nm}$, respectively.

The motivation for this paper was to demonstrate the utility of telescope-assisted hyperspectral measurements through indoor (laboratory) and outdoor (field) collection using different soil specimens as targets. This paper presents the methods used to collect data and a related discussion of the results. Additionally, the signal noise of the measurements is characterized as a function of wavelength across the entire receiver spectrum, and future improvements to the SOLAS receiving system are suggested.

\section{Methods}

The SOLAS receiver was tested in both indoor and outdoor environments at various ranges from the target. Proximal range measurements performed both indoors and outdoors were also collected to provide comparisons for the telescope-assisted measurements. The methods that were used to collect the hyperspectral data are described.

\subsection{Indoor and Outdoor Proximal Range Data Collection}

Specimens consisting of five different soil materials (Ottawa sand, coarse river sand, Donna Fill, bentonite soil, and kaolinite soil) were characterized in the laboratory using a benchtop setup. An 8-deg field-of-view (FOV) fore optic was attached to the end of the fiber-optic bundle leading to the ASD FieldSpec 4 spectroradiometer. The setup, as shown in Fig. 1, was similar to that used by other researchers ${ }^{13}$ and as recommended by ASD for conventional measurements in the

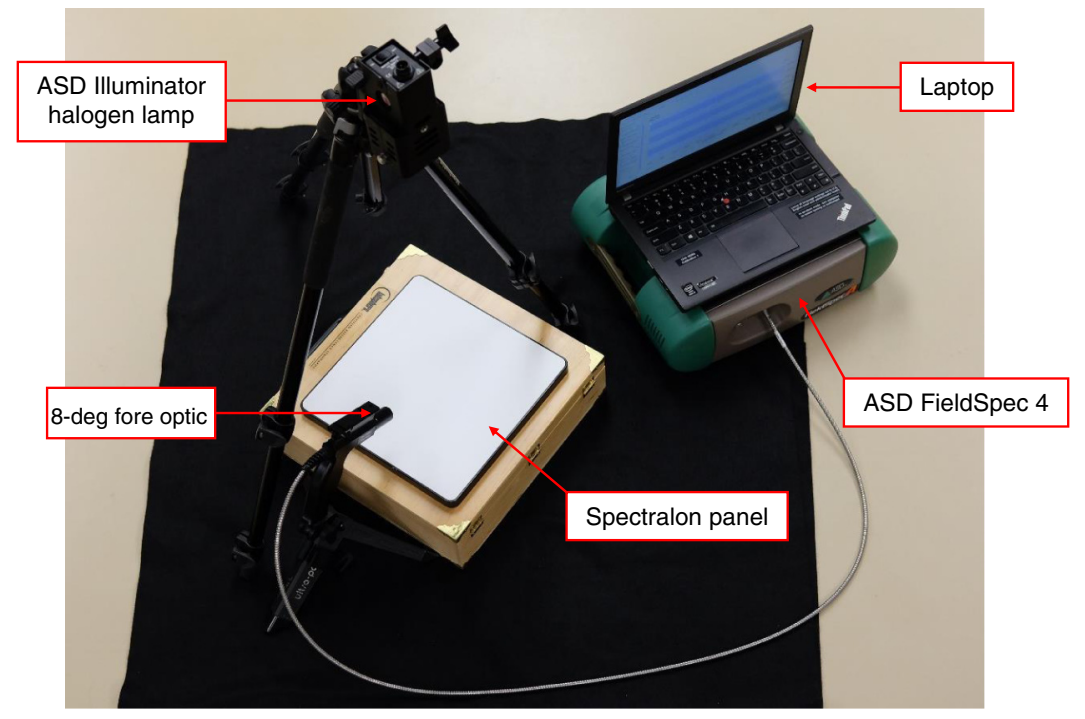

Fig. 1 Labeled photograph of the laboratory setup as used to collect proximal range spectral reflectance measurements with the ASD FieldSpec $4 \mathrm{Hi}$-Res spectroradiometer (pictured with calibrated Spectralon ${ }^{\circledR}$ reference panel as target). 
laboratory or field. A $25 \times 25 \mathrm{~cm}^{2}$ calibrated reference panel (Spectralon ${ }^{\circledR}$; Labsphere Inc., North Sutton, New Hampshire) was placed in the FOV of the fore optic. For proximal near-field range $(<1 \mathrm{~m})$ measurements, the perpendicular diameter of the FOV $D_{\mathrm{FOV}}$ was determined using Eq. (1).

$$
D_{\mathrm{FOV}}=D_{\mathrm{F} . \mathrm{O} .}+2 \cdot R \cdot \tan \left(\frac{\theta}{2}\right)
$$

where $D_{\mathrm{F} . \mathrm{O}}$ is the diameter of the fore optic lens, $R$ is the range, and $\theta$ is the angular FOV in degrees. The perpendicular $D_{\mathrm{FOV}}$ for the aforementioned laboratory setup corresponded with the perpendicular $D_{\mathrm{FOV}}$ for the SOLAS receiver for ranges of 20,35,50,60, and $100 \mathrm{~m}$. The distance between the fore optic and the target surface, equivalent to the $D_{\mathrm{FOV}}$ at a given range in the field, was determined using the relationship presented in Eq. (1).

The incidence angle of the fore optic relative to the target surface was set to $30 \mathrm{deg}$ to match typical field scale measurements performed using the SOLAS. A tungsten quartz, full-spectrum, halogen lamp (ASD "Illuminator") was directed perpendicular to the target surface. The FieldSpec 4 instrument and Illuminator lamp were allowed to warm up for $1 \mathrm{~h}$ before any spectra were acquired to minimize temperature-induced radiometric errors. The following procedure was repeated for each set of measurements, for each $D_{\text {FOV }}$ equivalent range. The fore optic was centered on the Spectralon panel, the instrument settings were optimized within the ASD RS ${ }^{3}$ software, and ten reference spectra were collected for a given acquisition. After referencing the Spectralon panel, the panel was successively replaced with one of seven 25-cm diameter, dry soil specimens (Ottawa sand, coarse river sand, Donna Fill with smooth and rough surface textures, bentonite soil, or kaolinite soil with smooth and rough surface textures). Ten spectra were collected for each specimen. A summary of the baseline measurements acquired for the soil specimens, collected from proximal range distances while indoors, is presented in Fig. 2.

The five soil types were also tested outdoors under solar illumination. The aforementioned procedures were repeated to collect reflectance spectra at a proximal range of $12 \mathrm{~cm}$ with an incidence angle of $44 \mathrm{deg}$ and an illumination angle of $\sim 60$ deg relative to the target surface. The $D_{\text {FOV }}$ was $2.4 \mathrm{~cm}$, which is equivalent to a SOLAS measurement at $40 \mathrm{~m}$ from the target. The resulting spectra served as a comparison for the SOLAS measurements that are described in Sec. 2.2. A summary of the measurements acquired for the soil specimens, collected from proximal range while outdoors, is presented in Fig. 3.

The tested specimens were selected because of previous studies in which benchmark characteristics were determined for the soils. ${ }^{13} \mathrm{~A}$ brief description of each of the soil types follows.

\begin{tabular}{|c|c|c|c|c|c|c|}
\hline $\begin{array}{c}\text { Acquisition } \\
\text { number }\end{array}$ & Target & $\begin{array}{c}\text { Incidence } \\
\text { angle (deg) }\end{array}$ & $\begin{array}{l}\text { Illumination } \\
\text { angle (deg) }\end{array}$ & $\begin{array}{c}\text { Range } \\
(\mathbf{c m})\end{array}$ & $\begin{array}{c}\text { Corresponding } \\
\text { range (m) }\end{array}$ & $\begin{array}{l}D_{\text {Fov }} \\
(\mathbf{c m})\end{array}$ \\
\hline \multirow[t]{2}{*}{$1,9,17,25,33$} & Spectralon ${ }^{\circledR}$ & & & 3 & 20 & 1.2 \\
\hline & & & & 10 & 35 & 2.1 \\
\hline \multirow[t]{2}{*}{$2,10,18,26,34$} & Ottawa sand & 30 & 90 & 16 & 50 & 3.1 \\
\hline & & & & 21 & 60 & 3.7 \\
\hline $3,11,19,27,35$ & Coarse sand & & & 38 & 100 & 6.1 \\
\hline $4,12,20,28,36$ & Donna Fill (smooth) & & & & & \\
\hline $5,13,21,29,37$ & Donna Fill (rough) & & & & $\begin{array}{l}\text { tificial } \\
\text { ination }\end{array}$ & \\
\hline $6,14,22,30,38$ & Bentonite & & & & & \\
\hline $7,15,23,31,39$ & Kaolinite (smooth) & & $30^{\circ}$ & & $90^{\circ}$ & \\
\hline $8,16,24,32,40$ & Kaolinite (rough) & & & & Iarget & \\
\hline
\end{tabular}

Fig. 2 Summary of measurements acquired indoors at different proximal ranges under artificial illumination. 


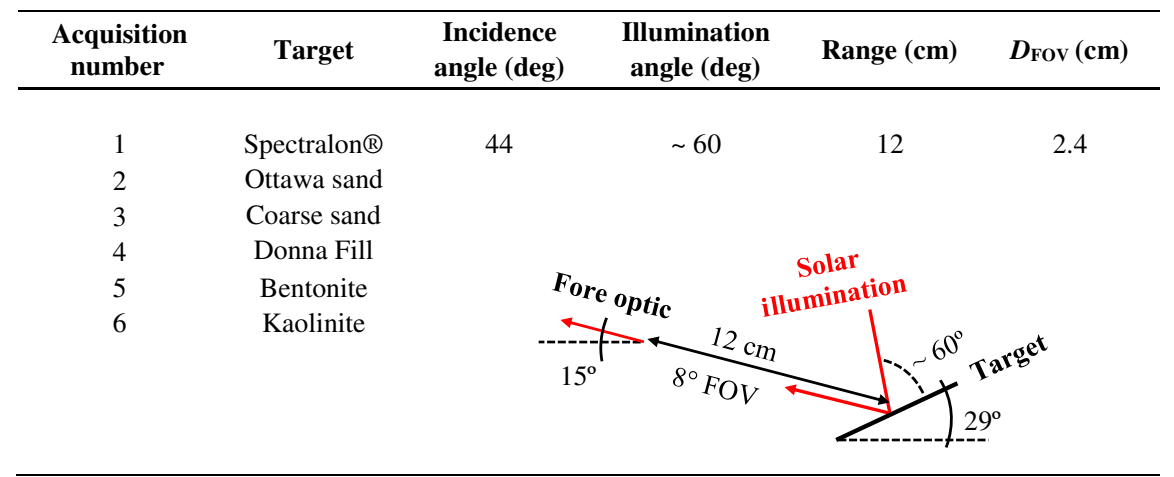

Fig. 3 Summary of measurements acquired outdoors at proximal range under solar illumination.

The Ottawa sand (Humboldt Mfg. Co., Elgin, Illinois) was a pure silica sand. The coarse, quartzitic river sand was sourced from the Arkansas River (Arkhola, Van Buren, Arkansas). The Donna Fill (Donna Fill Co., Little Rock, Arkansas) was a synthetic nepheline synetite material that was dark gray in color. The bentonite soil was a tan-colored smectitic clay (PondSeal ${ }^{\mathrm{TM}}$ ) that was sourced from Wyoming. The kaolinite soil was a commercial KaoWhite-S product (Thiele Kaolin Co., Sandersville, Georgia).

\subsection{Outdoor Intermediate-Range Data Collection}

The SOLAS receiver was tested in an outdoor environment at the Cato Springs Research Center (CSRC) that is located south of the University of Arkansas campus. The SOLAS device was set up on the rooftop of the CSRC, as depicted in Fig. 4, to allow for a better vantage point and a greater incidence angle relative to the target. The angular FOV of the SOLAS receiver was $3.5 \times 10^{-2} \mathrm{deg}$. The range-dependent $D_{\mathrm{FOV}}$ of the SOLAS receiver was previously described in more detail. ${ }^{12}$ Each specimen was $25 \mathrm{~cm}$ in diameter and was prepared dry. The same five soil types that were tested proximally were tested at a range of $40 \mathrm{~m}$. The target was inclined $29 \mathrm{deg}$ relative to the ground, while the SOLAS receiver was declined $15 \mathrm{deg}$ for an effective incidence

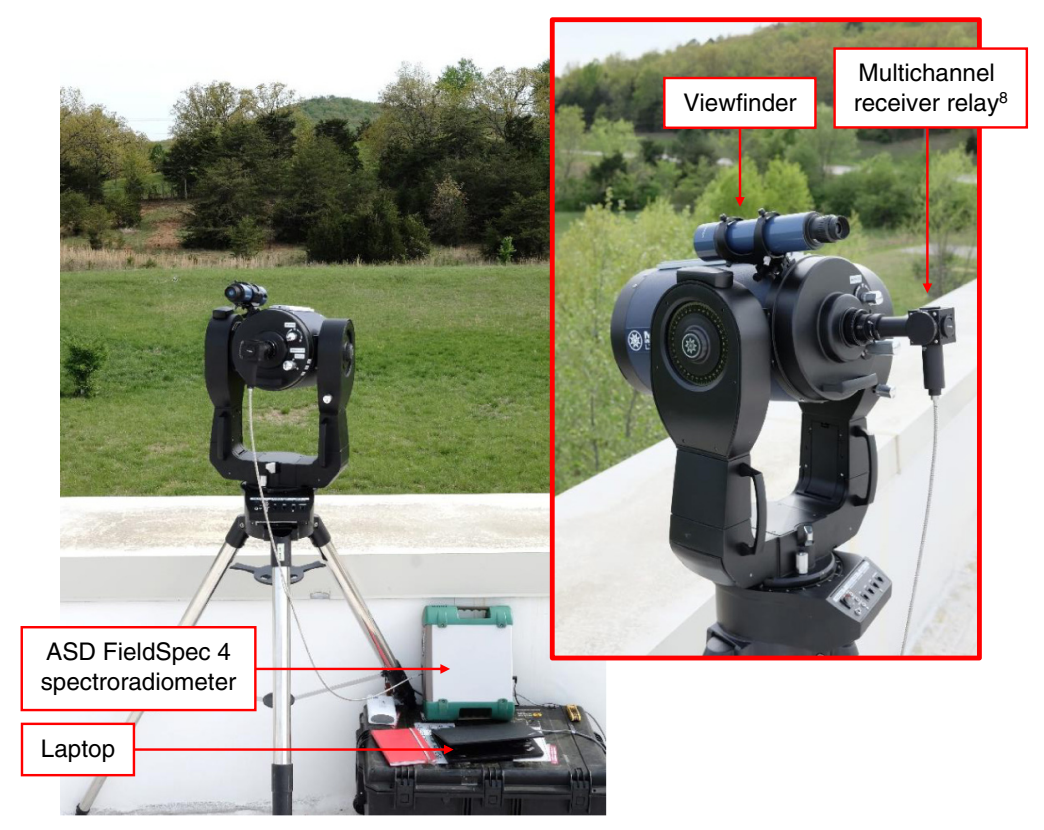

Fig. 4 Labeled photograph of the field setup as used to collect intermediate-range spectral reflectance measurements with the SOLAS telescope-enhanced receiver. 


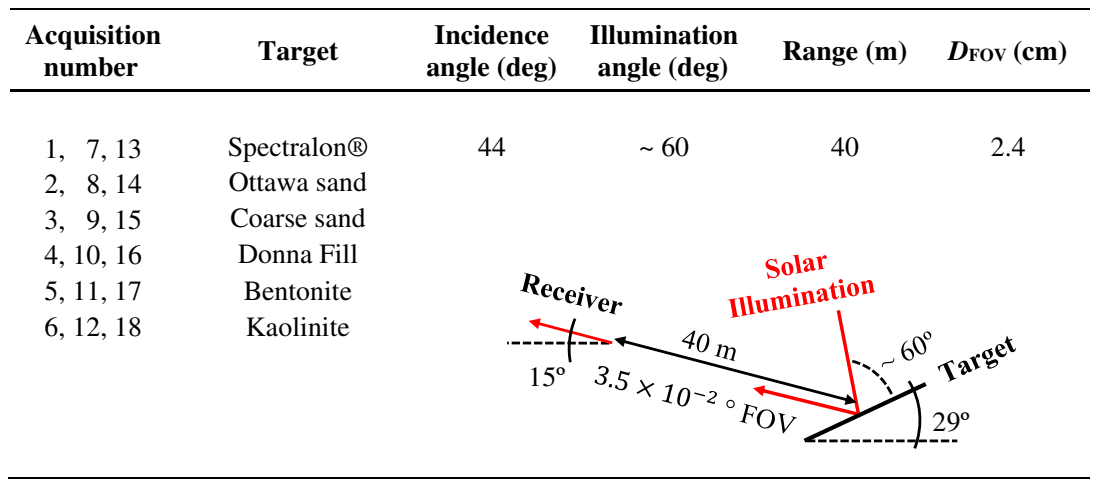

Fig. 5 Summary of measurements acquired outdoors at a range of $40 \mathrm{~m}$ under solar illumination.

angle of 44 deg relative to the target. The illumination angle from the sun was approximated based on the time of year and time of day using the spherical trigonometric relationship presented in Eq. (2).

$$
\cos \left(\theta_{s}\right)=\sin (\Phi) \cdot \sin (\delta)+\cos (\Phi) \cdot \cos (\delta) \cdot \cos (h),
$$

where $\theta_{s}$ is the solar zenith angle, $\Phi$ is the local latitude, $\delta$ is the sun declination, and $h$ is the hour angle (local solar time). During the readings, the sky was clear (no cloud coverage) and the air temperature was $22^{\circ} \mathrm{C}$ with a humidity of $43 \%$ and a wind speed of $20 \mathrm{~km} \mathrm{~h}^{-1}$.

To determine the repeatability of the measurements under outdoor conditions and solar illumination, the set of measurements was repeated twice within $30 \mathrm{~min}$. As described in Sec. 2.1, the set of specimens was also tested at the proximal range of $12 \mathrm{~cm}$ with the aforementioned 8-deg fore optic under the same solar illumination conditions. These proximal measurements provided a comparison to the outdoor measurements collected at a range of $40 \mathrm{~m}$. A summary of the intermediate-range measurements as acquired outdoors at a range of $40 \mathrm{~m}$ is presented in Fig. 5 .

\subsection{Processing Methods}

For each set of measurements of a given specimen, the raw reflectance values were averaged and normalized with respect to the Spectralon reference panel. The resulting reflectance values were then plotted as a function of wavelength. While the proximal range measurements did not require additional corrections, the SOLAS telescope-assisted measurements collected indoors under artificial illumination ${ }^{12}$ were splice corrected ${ }^{14}$ if an offset was observed at the transition wavelengths between the three instrument detector ranges (VNIR, SWIR 1, and SWIR 2). For the measurements collected outdoors under solar illumination, the 1900-nm long-path water vapor absorption band interfered with the aforementioned splice correction procedure at the 1800-nm detector transition. Therefore, the spectra collected outdoors were not corrected. A Savitzky-Golay filter ${ }^{15}$ was applied to smooth all of the collected spectra except for the indoor proximal range measurements, which did not require filtering.

For each of the aforementioned sets of measurements, the noise was characterized. A procedure outlined by $\mathrm{ASD}^{16,17}$ was followed to determine the baseline noise of the detectors within the FieldSpec 4 instrument. The noise-equivalent radiance was determined indoors at a proximal range using the Spectralon reference panel as the target due to the Lambertian reflectance properties of the panel across the 350- to 2500- nm range. The noise-equivalent radiance was used to derive the signal-to-noise ratio (SNR) across the range of wavelengths for typical measurements (1) performed at proximal ranges using the 8-deg fore optic indoors and outdoors and (2) collected at various intermediate ranges using the SOLAS telescope-enhanced receiver indoors and outdoors. 


\section{Results and Discussion}

\subsection{Indoor Proximal Range Measurements}

The baseline spectra for each of the five materials that were tested in this study are presented in Fig. 6(a). Because the measurements were collected indoors at proximal ranges and under artificial illumination, as described in Sec. 2.1, the resulting spectra were expected to be smooth and contain minimal noise. The spectra are presented in terms of absolute reflectance with respect to wavelength to allow for comparison with the United States Geological Survey (USGS) spectral library data. ${ }^{18}$ The spectra for the Ottawa sand [Fig. 6(b)], bentonite soil [Fig. 6(c)], and kaolinite soil [Fig. 6(d)] specimens generally matched the USGS standards. The USGS spectral library did not contain a material called "bentonite." The closest match within the database was a montmorillonite clay soil. Although both soils are in the Smectite group and share mineralogical properties, it is likely that the color of the soil and perhaps the particle size distribution differed. Moreover, the USGS reflectance spectra were collected with similar though different ASD spectroradiometers and with a perpendicular incidence angle.

As shown in Fig. 6(d), the surface roughness had an influence on the amplitude of the reflectance values, while the general trends including absorption features were well preserved. The kaolinite soil specimen that was prepared with a smooth surface texture reflected more light across the entire VNIR and SWIR wavelength range than the kaolinite soil specimen that

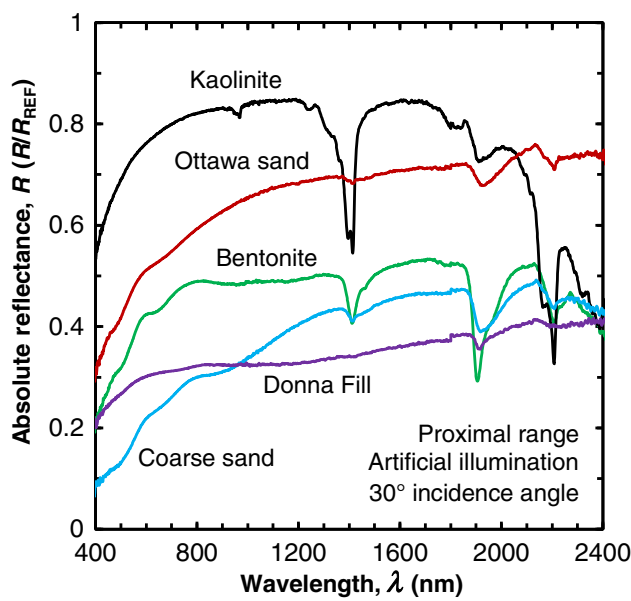

(a)

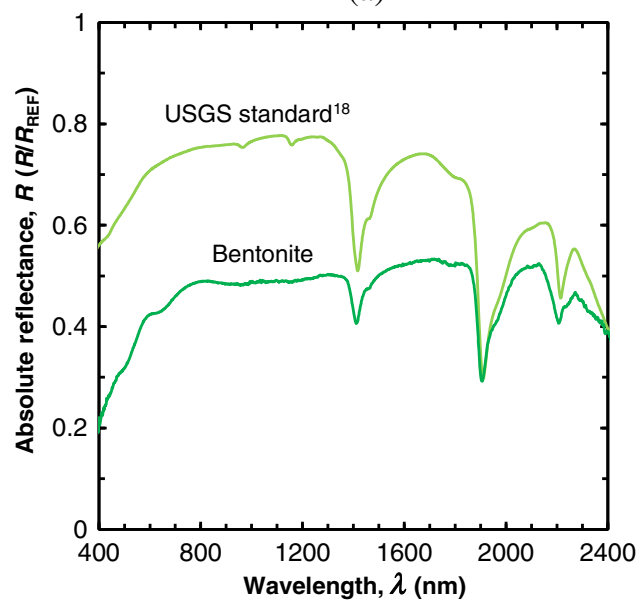

(c)

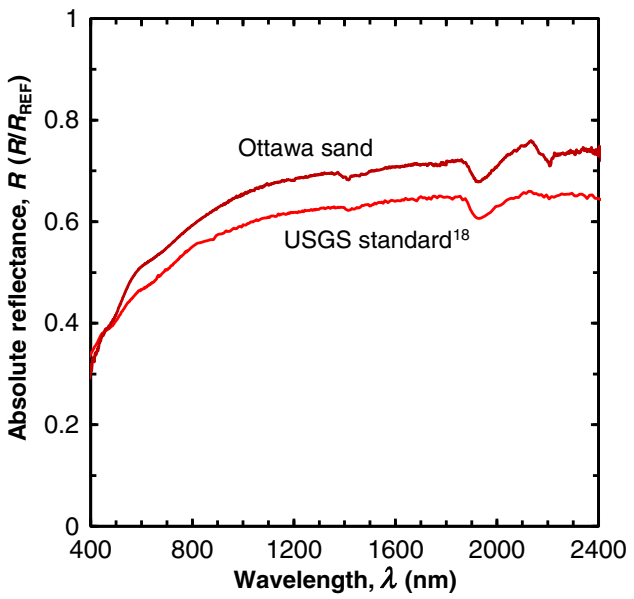

(b)

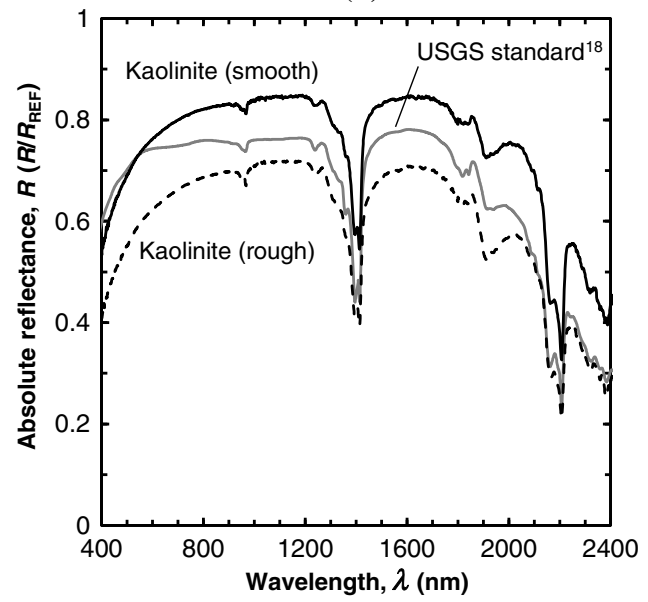

(d)

Fig. 6 Absolute reflectance spectra for dry specimens of (a) five soil types collected indoors at proximal ranges, (b) Ottawa sand, (c) bentonite soil, and (d) kaolinite soil, as compared with the USGS spectral library standards. ${ }^{18}$ 
was prepared with a rough surface texture. A similar, though less pronounced, effect was observed for the darker Donna Fill specimens that were prepared with smooth and rough surface textures.

Another factor that affected the spectral amplitude was the range-dependent FOV diameter $D_{\text {FOV }}$ or spatial resolution of the measurements. An increase in $D_{\text {FOV }}$ generally resulted in an increase in the reflectance values across the wavelength range for the rough-textured kaolinite soil and Donna Fill specimens, as shown in Figs. 7(a) and 7(b), respectively. This was due to more light being reflected into the fore optic, given an increase in the distance between the fore optic and the target surface. For $D_{\text {FOV }}>6 \mathrm{~cm}$, the effect of range on the spectral amplitude was negligible. Likewise, the effect was insignificant for the other soil specimens, including the smooth-textured kaolinite and Donna Fill specimens (not shown in Fig. 7).

\subsection{Outdoor Intermediate Range Measurements}

The results for the outdoor intermediate-range measurements that were described in Sec. 2.2 are shown in Figs. 8 and 9. The repeatability of the measurements is demonstrated in Fig. 8. The average of the three measurements, acquired for each specimen using the SOLAS receiver at a range of $40 \mathrm{~m}$, is shown in Fig. 9 alongside the indoor and outdoor proximal measurements. When comparing the telescope-assisted measurements to the spectra collected proximally, the $D_{\text {FOV }}$ was matched as closely as possible though the range-dependent $D_{\text {FOV }}$ effect was small, as discussed in Sec. 3.1. The indoor and outdoor measurements compared well. The outdoor measurements, acquired under solar illumination, contained a loss in SNR around the 1400- and 1900-nm bands, typical of long-path atmospheric water vapor absorption. The erroneous reflectance values around these bands were preserved to illustrate the effect on the signal; however, they are commonly removed in postprocessing. As described in Sec. 2.3, the transition between adjacent detectors in the FieldSpec instrument sometimes resulted in an offset that was addressed with a simple splice correction procedure during processing. However, this procedure was not applicable to the transition between the two SWIR detectors because of the interference of the long-path water vapor absorption band.

Spectral signatures outside of the long-path absorption bands were identifiable. For example, the kaolinite spectra contained the characteristic doublet feature in the 2200-nm region, as presented in Fig. 10, and the bentonite spectra retained the signature in the 2200 to $2300 \mathrm{~nm}$ region. The Ottawa and river sands contained weak features with signature peaks at $2140 \mathrm{~nm}$ followed

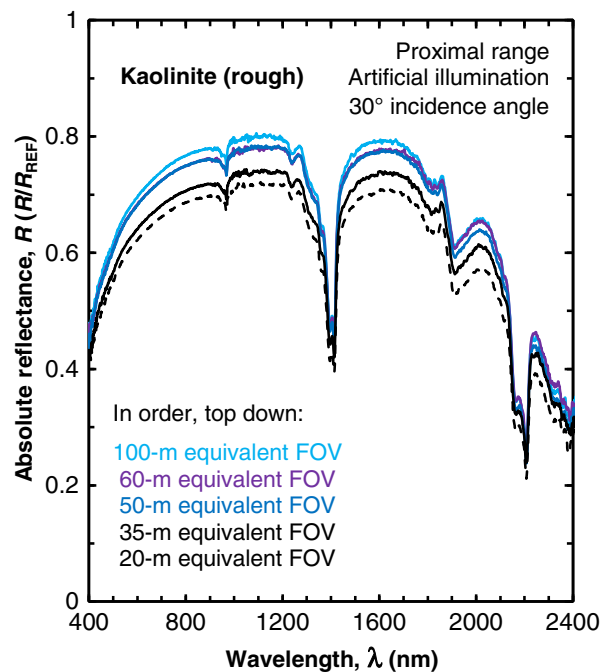

(a)

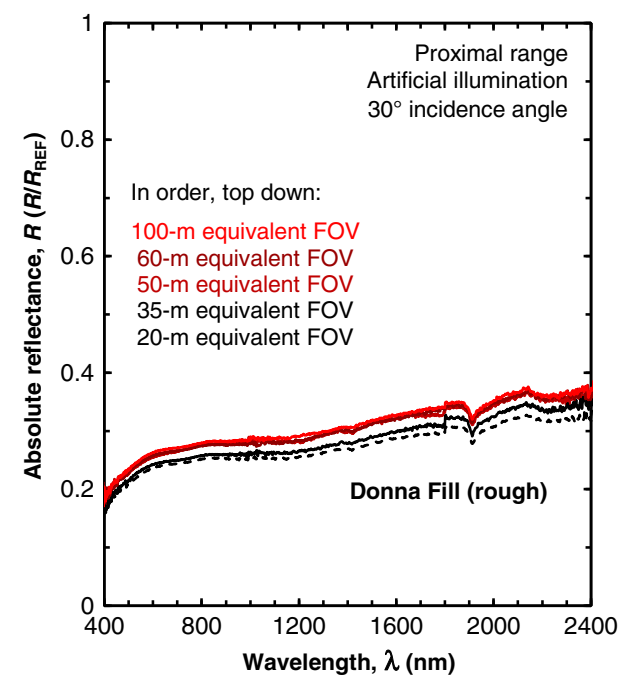

(b)

Fig. 7 Absolute reflectance spectra for dry specimens of (a) kaolinite soil with a rough surface texture and (b) Donna Fill with a rough surface texture, collected indoors at proximal ranges corresponding to equivalent FOV diameters as observed with the SOLAS at distances of $20,35,50$, 60 , and $100 \mathrm{~m}$ from the target. 


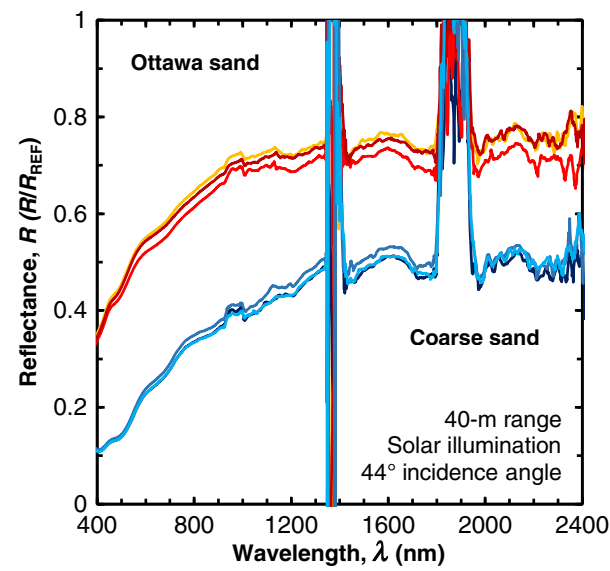

(a)

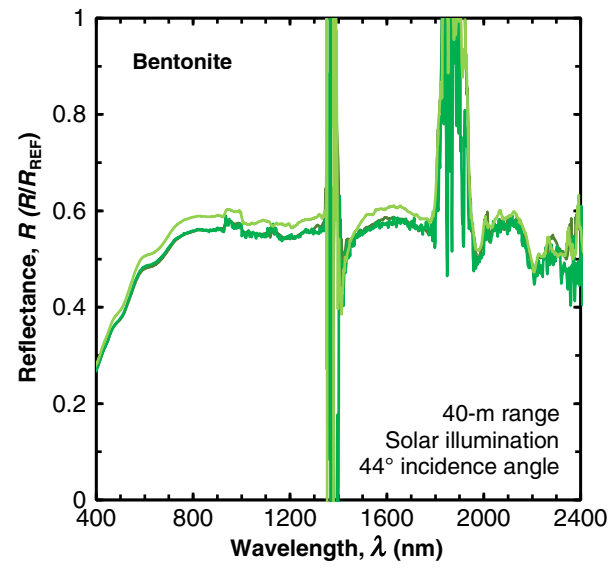

(c)

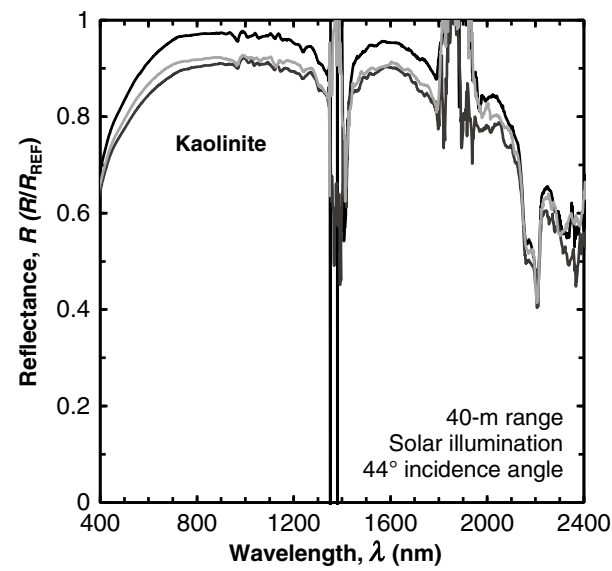

(b)

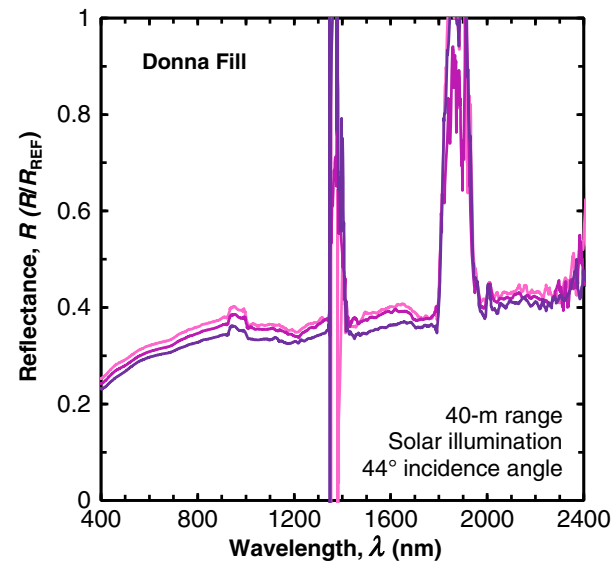

(d)

Fig. 8 Three independent sets of reflectance spectra acquired outdoors under solar illumination over a period of $30 \mathrm{~min}$ at a range of $40 \mathrm{~m}$ for dry specimens of (a) Ottawa sand and coarse river sand, (b) kaolinite soil, (c) bentonite soil, and (d) Donna Fill.

by troughs at $2200 \mathrm{~nm}$. As expected, the Donna Fill spectra contained no distinguishable features.

The spectra for all of the specimens collected with the SOLAS receiver contained a feature around the 970-nm water absorption band; however, the absorption feature was only discernible for the kaolinite spectra. An inverted artifact with increased reflectivity was observed for the sand, bentonite, and Donna Fill specimens. It is hypothesized that the decreased sensitivity at the upper range of the VNIR detector and the lower range of the SWIR 1 detector, as discussed in Sec. 3.4, contributed to the artifact, with the possibility of the telescope amplifying the effect.

\subsection{Noise-Equivalent-Radiance Characterization}

The noise statistics for typical telescope-assisted measurements were presented previously; ${ }^{12}$ however, the noise was not previously characterized across the entire VNIR to SWIR wavelength range. Therefore, for completeness, the noise-equivalent radiance for the ASD FieldSpec 4 instrument is plotted as a function of wavelength in Fig. 11(a). For each of the three detectors, the measured noise was greatest near the edges of each detector range, which is attributed to reduced sensitivity of the silicon and InGaAs detectors at these wavelengths. ${ }^{16,19}$ While the SWIR 1 range (1001 to $1800 \mathrm{~nm}$ ) was the most stable range statistically, it also contained the greatest noise-equivalent-radiance value $\left(7.5 \times 10^{-9} \mathrm{~W} \mathrm{~cm}^{-2} \mathrm{~nm}^{-1} \mathrm{sr}^{-1}\right.$ at $\left.1001 \mathrm{~nm}\right)$.

As described in Sec. 2.3, the noise-equivalent radiance was used to derive the SNR across the entire range of collected wavelengths. A plot of the SNR for typical indoor and outdoor 


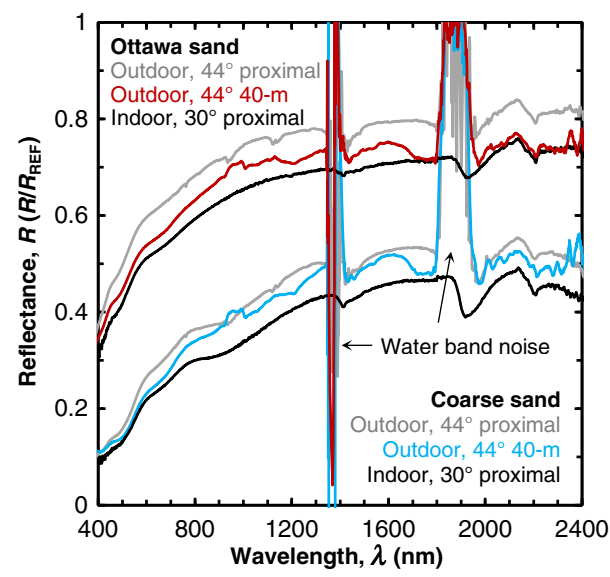

(a)

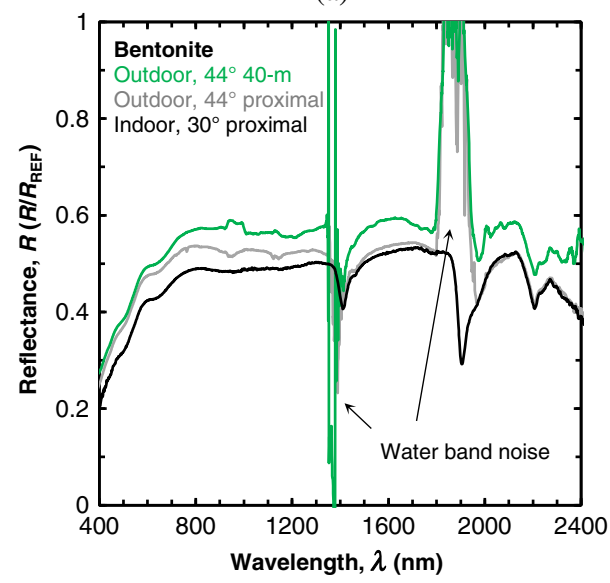

(c)

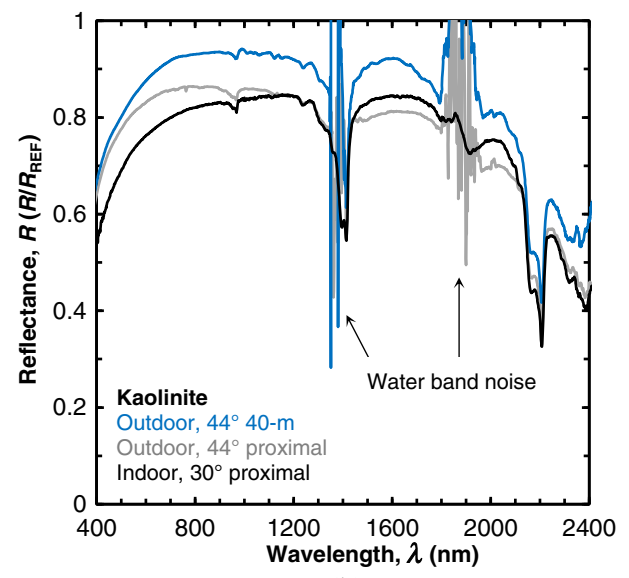

(b)

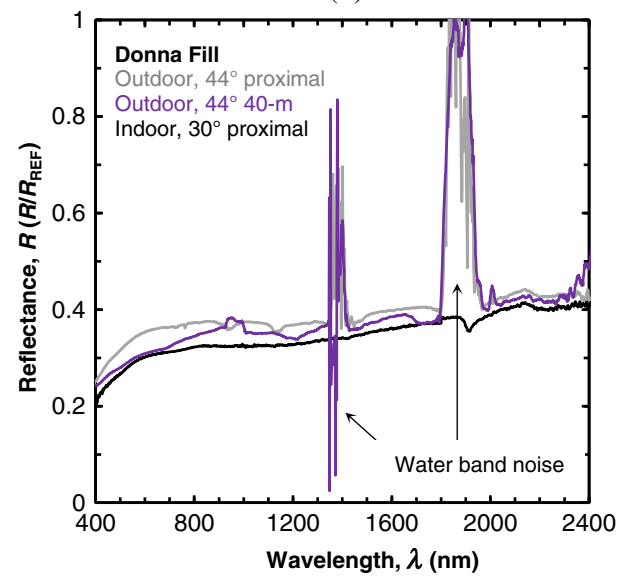

(d)

Fig. 9 Reflectance spectra acquired indoors and outdoors at proximal ranges and outdoors at a range of $40 \mathrm{~m}$ for dry specimens of (a) Ottawa sand and coarse river sand, (b) kaolinite soil, (c) bentonite soil, and (d) Donna Fill.

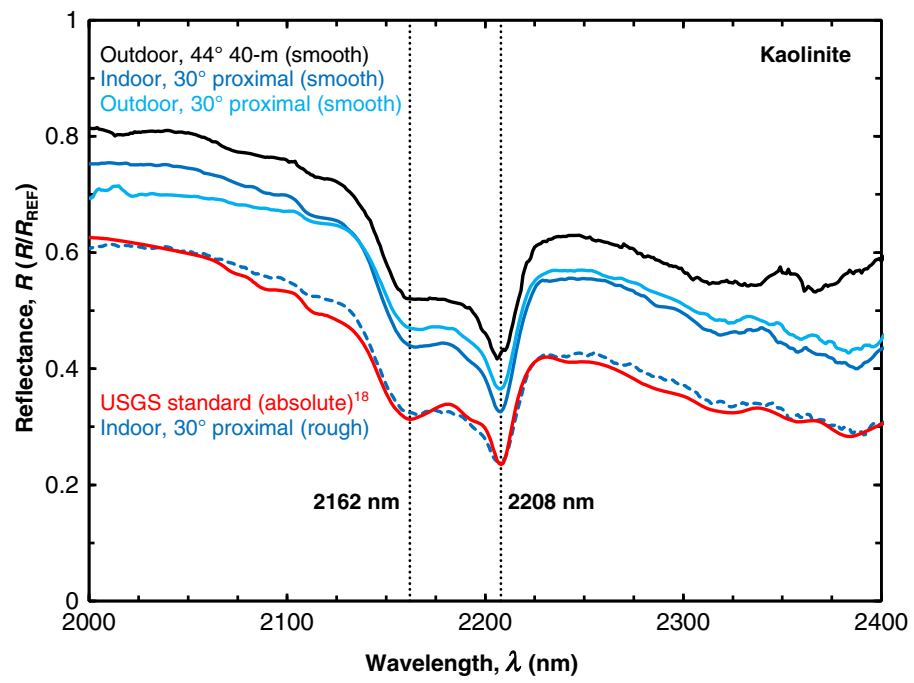

Fig. 10 Reflectance spectra acquired for kaolinite soil centered around the characteristic doublet feature acquired indoors and outdoors at proximal range and outdoors at a range of $40 \mathrm{~m}$ as compared with the USGS spectral library standard..$^{18}$ 


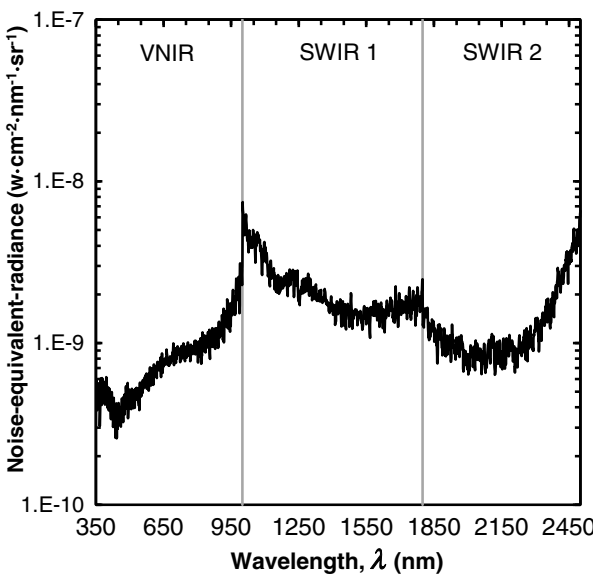

(a)

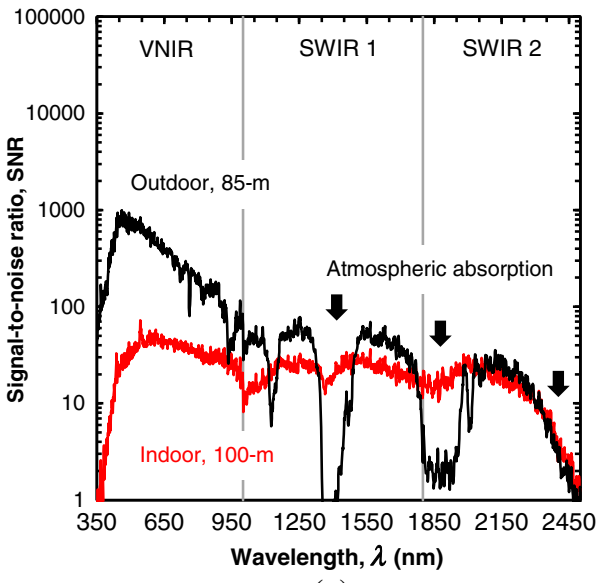

(c)

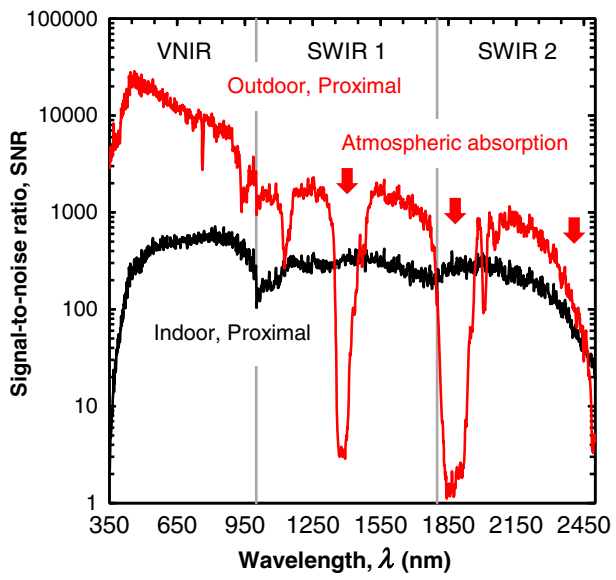

(b)

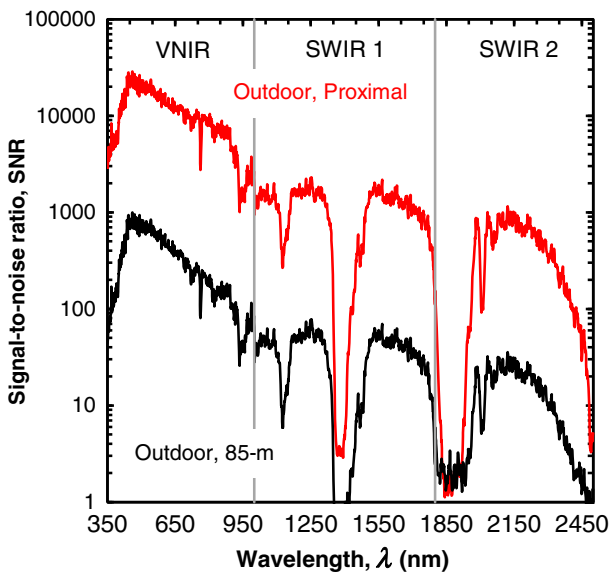

(d)

Fig. 11 (a) Noise as a function of wavelength as measured across the three detector ranges of the ASD FieldSpec 4 spectroradiometer (one VNIR and two SWIR detectors) and typical SNRs as functions of wavelength for (b) indoor and outdoor measurements acquired at proximal range, (c) indoor and outdoor telescope-assisted measurements acquired at ranges of 100 and $85 \mathrm{~m}$, respectively, and (d) outdoor measurements acquired at a proximal range of $85 \mathrm{~m}$ (telescopeassisted).

measurements, as acquired at proximal range, is presented in Fig. 11(b). The measurements acquired under solar illumination had a greater SNR than the measurements acquired under artificial illumination, except for where the sunlight was absorbed by the atmosphere. Similarly, as presented in Fig. 11(c), a comparison of the SNR for telescope-assisted, remote measurements performed indoors and outdoors indicated that the SNR was greater for the outdoor measurements. A comparison of proximal and remote measurements acquired outdoors under solar illumination revealed far greater SNR for the proximal measurements, as presented in Fig. 11(d).

According to previous studies using an ASD FieldSpec 4 instrument, ${ }^{19}$ increased levels of noise were observed due to ambient temperature fluctuations and different sensor radiance levels. The noise manifested itself at the edges of each of the three detector wavelength ranges in the form of radiometric "jumps" between detectors; however, the noise was always greatest at the far edges of the instrument range (near 350 and $2500 \mathrm{~nm}$ ). The ambient temperature-induced noise was minimized once the instrument had achieved internal thermal equilibrium requiring a 1-h (minimum) warm-up period. Ambient temperature fluctuations of $5^{\circ} \mathrm{C}$ or more also required time to achieve thermal equilibrium within the instrument. It is recommended that future measurements be collected in radiance mode (as opposed to reflectance mode). This will allow for the application of a parabolic correction algorithm (available in the ASD software) before calculating reflectance factors for further analysis. Corrections that were applied directly to the 
reflectance data did not fully address the thermal noise nor were the corrections applicable to measurements that were affected by long-path water vapor absorption near the 1900-nm water band (as discussed in Sec. 3.3).

\subsection{Limitations and Future Applications}

The measurements that were collected for this study were limited by several factors. For example, the maximum incidence angle of the reflectance measurements was limited primarily by the angle of repose of the dry granular soil specimens (Ottawa and coarse river sands). Despite this constraint, it was demonstrated that the SOLAS receiver could be utilized to collect measurements from oblique targets that mimicked soil slopes and other natural surfaces. Another major limitation for testing the maximum range of the SOLAS receiver was the size of the Spectralon reference panel used in this study. Specifically, the range-dependent $D_{\mathrm{FOV}}$ (spot size) of the receiver could not exceed the elevation (effective height) of the target, which was significantly reduced by the shallow incidence angles tested in this study. Conversely, the azimuthal dimension of the FOV was not affected by the incidence angle.

A solution to the target size limitation is the incorporation of a companion spectrometer that measures a reference simultaneous to the sample target (e.g., ASD FieldSpec Dual methodology). This would eliminate the need for placing a reference panel downrange making long-range measurements more practical. An added benefit of near-simultaneous referencing of the Spectralon panel is the reduction of error associated with rapid changes in atmospheric conditions (cloud cover and wind). Although the outdoor measurements presented in this study were collected under relatively stable atmospheric conditions, even small variations in the solar irradiance or illumination angle resulted in changes in the amplitude of the reflectance spectra and the SNR. Another solution to the range limitation due to the diverging FOV could be provided by modifying the receiver, which was described previously, ${ }^{12}$ to achieve a narrower FOV for more effective intermediate- and long-range measurements.

In the future, the SOLAS will incorporate differential laser absorption measurements. ${ }^{11}$ These measurements of atmospheric attenuation due to absorption and scattering by water vapor along the receiver path will enable corrections to determine exact reflectance measurements. Deployment of the SOLAS will enable remote classification of surface materials over large areas that are hazardous or otherwise difficult to access. Examples of the applications of the SOLAS include rapid characterization of rocks, minerals, soils, or vegetation on slopes or outcroppings for economic geology, agriculture or forestry research, monitoring of soils used in construction projects (e.g., mining operations and tailings dams), or forensic investigation of geohazards (e.g., landslides and debris flows). The SOLAS could also provide high spatial, spectral, and temporal resolution ground truth data for current multispectral (e.g., Sentinel- $2^{20}$ ) or future hyperspectral (e.g., EnMAP ${ }^{21}$ and HyspIRI ${ }^{22}$ ) satellite missions. More information about the SOLAS instrument is available in the literature. ${ }^{11,12}$

\section{Conclusions}

Hyperspectral reflectance measurements were acquired for five different soil types (Ottawa sand, coarse river sand, Donna Fill, bentonite soil, and kaolinite soil) under artificial illumination (indoors) and solar illumination (outdoors). Telescope-assisted measurements acquired using the SOLAS device were demonstrated for an intermediate range of $40 \mathrm{~m}$ from the target. Reflectance spectra acquired at a proximal range in the laboratory and the field were provided for comparison. The spectra matched well with distinguishable absorption and reflectance features characteristic of soil mineralogy. Additionally, the effect of specimen type, rangedependent spatial resolution, and environment on the measured reflectance was presented and discussed.

Adoption of the ASD FieldSpec 4 spectroradiometer instrument into the SOLAS platform reduced the typical SNR of measurements performed at the proximal range; however, an acceptable SNR was maintained for the telescope-assisted intermediate-range measurements. The SNR of the measurements was determined as a function of wavelength for the full spectral range of 
the receiver. The greatest overall SNR was observed for the proximal measurements that were performed outdoors, followed by the proximal measurements that were performed indoors, the remote measurements that were performed outside, and finally, the remote measurements that were performed indoors. The average ratio of the proximal range SNR to the intermediate-range SNR was 16:1. As expected, signal loss around the 1400- and 1900-nm bands due to long-path atmospheric water vapor absorption was observed for the outdoor measurements. Increased levels of noise, inherent to the design of the spectroradiometer, were observed for the wavelengths near the edges of each detector range $(350,1000,1800$, and $2500 \mathrm{~nm})$. Proper instrument warm-up practices minimized the radiometric noise present in the signal, particularly at the transition from one detector to the next. Future development of the SOLAS remote sensor will allow for derivation of exact reflectance measurements over longer ranges.

\section{Acknowledgments}

This project was funded by the U.S. Department of Transportation (USDOT) through the Office of the Assistant Secretary for Research and Technology (OST-R) under USDOT Cooperative Agreement No. OASRTRS-14-H-UARK. The views, opinions, findings, and conclusions reflected in this publication are solely those of the authors and do not represent the official policy or position of the USDOT/OST-R or any state or other entity. USDOT/OST-R does not endorse any third-party products or services that may be included in this publication. This material is also based upon work supported by the National Science Foundation Graduate Research Fellowship Program under Grant No. DGE-1450079. Any opinions, findings, and conclusions or recommendations expressed in this material are those of the authors and do not necessarily reflect the views of the National Science Foundation. The authors declare no conflicts of interest. The funders had no role in the design of the study, in the collection, analyses, or interpretation of data, in the writing of the paper, or in the decision to publish the results.

\section{References}

1. E. Adam, O. Mutanga, and D. Rugege, "Multispectral and hyperspectral remote sensing for identification and mapping of wetland vegetation: a review," Wetlands Ecol. Manag. 18, 281-296 (2010).

2. F. D. van der Meer et al., "Multi- and hyperspectral geologic remote sensing: a review," Int. J. Appl. Earth Obs. Geoinf. 14, 112-128 (2012).

3. B. K. Waruru et al., "Rapid estimation of soil engineering properties using diffuse reflectance near infrared spectroscopy," Biosyst. Eng. 121, 177-185 (2014).

4. E. Pasolli et al., "Advances in hyperspectral image classification methods for vegetation and agricultural cropland studies," in Hyperspectral Indices and Image Classifications for Agriculture and Vegetation, P. S. Thenkabail, J. G. Lyon, and A. Huete, Eds., 2nd ed., pp. 67-104, CRC Press, Boca Raton, Florida (2018).

5. A. F. H. Goetz, "Three decades of hyperspectral remote sensing of the Earth: a personal review," Remote Sens. Environ. 113, S5-S16 (2009).

6. M. E. Schaepman et al., "Earth system science related imaging spectroscopy-an assessment," Remote Sens. Environ. 113, S123-S137 (2009).

7. P. C. Pandey et al., "The use of hyperspectral earth observation data for land use/cover classification: Present status, challenges, and future outlook," in Advanced Applications in Remote Sensing of Agricultural Crops and Natural Vegetation, P. S. Thenkabail, J. G. Lyon, and A. Huete, Eds., 2nd ed., pp. 147-174, CRC Press, Boca Raton, Florida (2018).

8. J. Transon et al., "Survey of hyperspectral earth observation applications from space in the Sentinel-2 context," Remote Sens. 10(3), 157 (2018).

9. P. Koirala et al., "Real-time hyperspectral image processing for UAV applications, using HySpex Mjolnir-1024," Proc. SPIE 10198, 1019807 (2017).

10. G. Jiang et al., "Identification of iron-bearing minerals based on HySpex hyperspectral remote sensing data," J. Appl. Remote Sens. 13(4), 047501 (2019). 
11. S. E. Salazar, C. D. Garner, and R. A. Coffman, "Development of a multimode field deployable lidar instrument for topographic measurements of unsaturated soil properties: instrument description," Remote Sens. 11(3), 289 (2019).

12. S. E. Salazar and R. A. Coffman, "Multi-channel optical receiver for ground-based topographic hyperspectral remote sensing," Remote Sens. 11(5), 578 (2019).

13. C. D. Garner, "Development of a multiband remote sensing system for determination of unsaturated soil properties," PhD Dissertation, University of Arkansas, Fayetteville, Arkansas (2017).

14. M. Danner et al., "Spectral sampling with the ASD FieldSpec 4-theory, measurement, problems, interpretation," EnMAP Field Guides Technical Report, pp. 1-20, GFZ Data Services, Potsdam, Germany (2015).

15. A. Savitzky and M. J. E. Golay, "Smoothing and differentiation of data by simplified least squares procedures," Anal. Chem. 36(8), 1627-1639 (1964).

16. Analytical Spectral Devices, ASD Technical Guide, D. C. Hatchell, Ed., 3rd ed., pp. 1-132, Analytical Spectral Devices, Inc., Boulder, Colorado (1999).

17. G. Fager, Analytical Spectral Devices, Longmont, Colorado, personal correspondence (2019).

18. R. F. Kokaly et al., USGS Spectral Library Version 7: U.S. Geological Survey Data Series 1035, pp. 1-61, United States Geological Survey, Reston, Virginia (2017).

19. A. Hueni and A. Bialek, "Cause, effect, and correction of field spectroradiometer interchannel radiometric steps," IEEE J. Sel. Top. Appl. Earth Obs. Remote Sens. 10(4), 1542-1551 (2017).

20. M. Drusch et al., "Sentinel-2: ESA's optical high-resolution mission for GMES operational services," Remote Sens. Environ. 120, 25-36 (2012).

21. L. Guanter et al., "The EnMAP spaceborne imaging spectroscopy mission for earth observation," Remote Sens. 7(7), 8830-8857 (2015).

22. HyspIRI Mission Concept Team, HyspIRI Final Report, pp. 1-87, Jet Propulsion Laboratory, California Institute of Technology, Pasadena, California (2018).

Sean E. Salazar is an engineer at the Norwegian Geotechnical Institute, Oslo. He received his BS, MS, and PhD degrees in civil engineering from the University of Arkansas in 2013, 2017, and 2019, respectively. His research focuses on applied remote sensing methods for natural and anthropogenic hazards.

Richard A. Coffman is an associate professor of civil engineering (geotechnical emphasis) at the University of Arkansas. He received his BS degree in civil engineering from the University of Wyoming in 2002, MS degree in civil engineering from the University of Texas at Austin in 2003, and PhD in civil engineering from the University of Missouri, Columbia, in 2009. His research focuses on soil behavior and remote sensing applications within geotechnical engineering. 